\title{
NAGELE, UN CONTINUO LABORATORIO URBANO Reinterpretando el proyecto urbano, hoy
}

\author{
Nagele, an urban lab in progress \\ Reviewing the urban plan nowadays
}

\author{
Abad Monllor, Enrique; \\ (Departamento de Cultura, CTAA), kike@enredad.com \\ Palacios Labrador, Luis
}

(Departamento de Proyectos Arquitectónicos, Grupo de investigación Cultura del Hábitat, ETSAM)

luispalacioslabrador@gmail.com

\section{RESUMEN}

La presente investigación supone la revisión crítica de la ciudad utópica de Nagele, asentamiento holandés ubicado en la nueva tierra del Noordoostpolder y diseñado de 1947 a 1955 por los grupos de arquitectos De 8 en Opbouw. Entre ellos, Gerrit Rietveld vislumbró el planeamiento de una ciudad acabada desde su origen y Aldo van Eyck la formuló como prototipo de su teoría de configuración urbana, hecho que ensalza el proceso de diseño urbano, objeto de esta investigación.

Revisitar Nagele 70 años después permite entender la transformación que ha sufrido a lo largo del tiempo debido a la crisis del modelo de explotación agrícola y la necesidad de nueva vivienda. Para ello, después de introducir la singularidad del diseño del todo, de las partes, y de su crecimiento, se lleva a cabo un análisis de la situación actual de la ciudad, para concluir con aportaciones recientes, llevadas a cabo desde ámbitos de investigación y docencia en Holanda y España, reinterpretando el proyecto en la actualidad.

A través del caso de estudio, la investigación aporta una reflexión sobre el futuro del patrimonio del urbanismo moderno de posguerra, abriendo el debate sobre su intención de mantener el diseño urbano original o permitir su reinterpretación, crecimiento y densificación atendiendo a las demandas agrícolas y residenciales de la ciudad contemporánea.

Palabras clave: Nagele, De 8 en Opbouw, Van Eyck.

Bloque temático: morfologías urbanas.

\begin{abstract}
This research proposes a critical review of Nagele, a Dutch settlement located in the new land of the Noordoostpolder and designed in the 50 s by the groups of architects De 8 en Opbouw. Among them, Gerrit Rietveld defined a plan unable to be expanded from its origin and Aldo van Eyck formulated Nagele as a prototype of his configurative theory; this fact praises the process of urban design, main goal of the research. Revisiting Nagele 70 years later allows understanding the transformation it has suffered over time due to the evolution of the agricultural model and the need for new housing. The research analyzes the singularity of the urban design of the whole and the parts, its growth and the current situation of the city. It concludes with recent contributions, carried out from different researching and teaching areas in the Netherlands and Spain. Throughout the case study, the research provides a discussion on the future of post-war modern urban heritage, the debate on its intention to either maintain the original urban design or allow its reinterpretation, growth and densification in response to the agricultural and residential demands of the contemporary city.
\end{abstract}

Keywords: Nagele, De 8 en Opbouw, Van Eyck.

Topic: urban morphologies. 


\section{Proceso de diseño}

\subsection{Evolución del planeamiento}

Heredada del movimiento artístico holandés De Stijl, la historia de Nagele estuvo marcada siempre por su carácter experimental. La ciudad fue diseñada ex novo para dos mil quinientas personas y sesenta hectáreas en el municipio de Noordoostpolder, un gran territorio ganado al mar. ${ }^{1}$ Su intención era acoger a los agricultores del campo neerlandés -propios de asentamientos dispersos- en una morfología urbana compacta, concéntrica y con entidad propia.

El planeamiento se desarrolló entre los años 1947 y 1956, un periodo de tiempo en que el discurso arquitectónico se encontraba en evolución en Europa, y en el cual Nagele participó activamente al exponerse en cuatro congresos CIAM consecutivos, desde su primera aparición en la reunión de Bérgamo de 1949. Durante la década que duró el diseño de planeamiento, fueron muchos los arquitectos que participaron en el proceso destacando, dentro de los grupos $D e 8$ en Opbouw, figuras reconocidas en el panorama arquitectónico holandés, como Rietveld, Van Eesteren, Van Eyck, Bakema, Stam, Ruys, etc. ${ }^{2}$
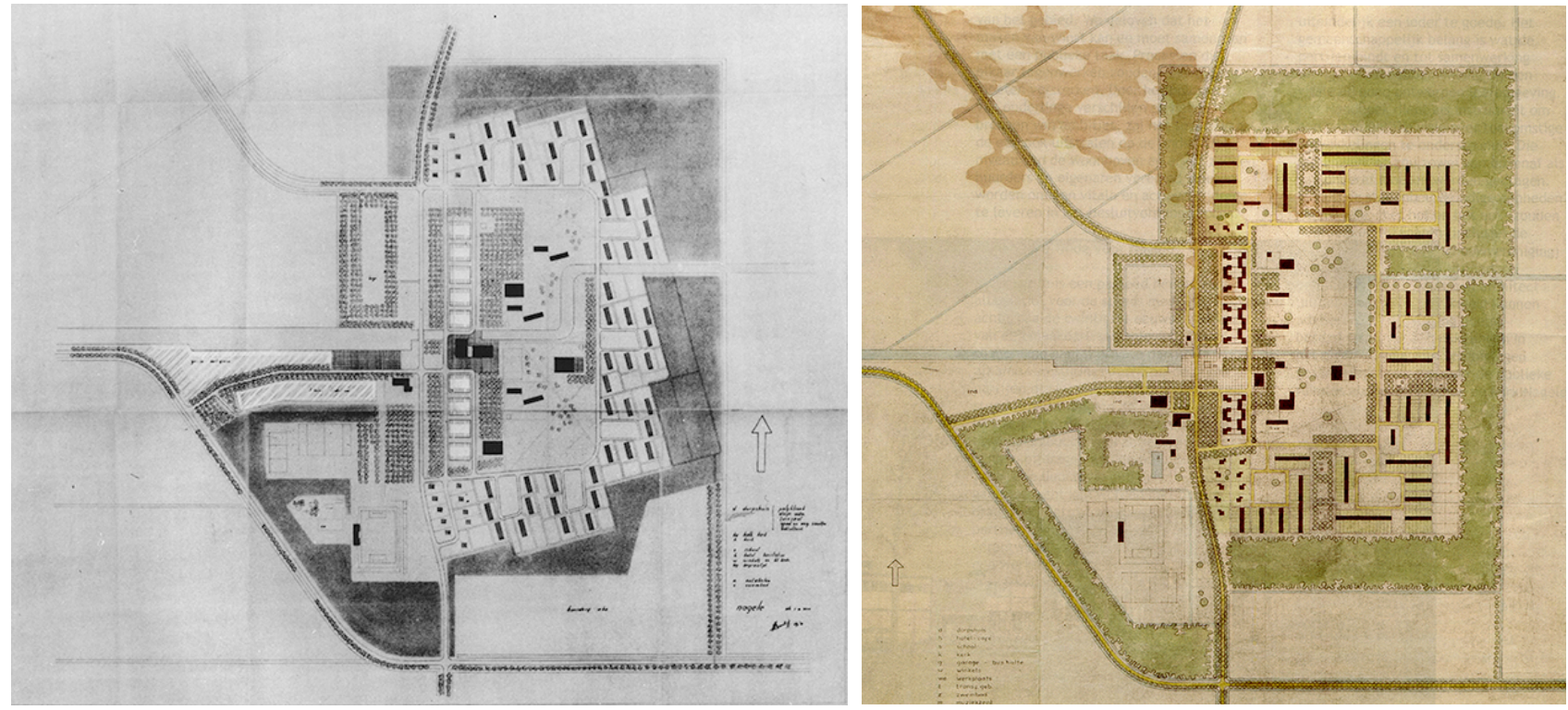

Fig. 01. (izq.) Proyecto para Nagele mostrado en el CIAM de Bérgamo, del 22 al 31 de julio de 1949. Kamerling, 17 diciembre de 1948. Fuente: (HEMEL, VAN ROSSEM, 1984)

Fig. 02 (dcha.) Plano de Aldo van Eyck para el 9 de marzo de 1954. Fuente: (V.V.A.A, Handboek Nagele. Visie op een modernistisch dorp in de polder, 2010)

En su primera etapa de diseño, Nagele se presentó como prototipo urbano funcionalista, ejemplo del planeamiento CIAM y la Carta de Atenas, que organizaba la ciudad higienista en la separación de las cuatro funciones básicas: trabajo, vivienda, movilidad y ocio (fig. 01). La segunda fase, poco antes de la desintegración de los CIAM y revisión del Team X de los principios del Movimiento Moderno, incorporó al proceso de diseño

\footnotetext{
${ }^{1}$ Ver: ABAD MONLLOR, Enrique. Nagele: un nuevo asentamiento en un paisaje artificial. Tesis Doctoral. Director: Emilia Hernández Pezzi, Francisco Arqués Soler. Universidad Politécnica de Madrid, Departamentos de Proyectos, ETSAM, 2016.

2 Los integrantes del grupo 'De 8' que se hicieron cargo del diseño de Nagele fueron: Merkelbach, Bodegraven, Bodon, Bijhouwer, Van Eesteren, Elling, Van Eyck, Gelderen, Kamerling, Kloos, Niegeman, Rietveld, Ruys, Salomonson, Stam, Vries. El grupo 'de 8' se encargó del primer diseño de Nagele (1947-49) y el grupo Opbouw se sumó al segundo diseño (1952-54), conociéndose el equipo redactor como 'de 8 en Opbouw'.
} 
estrategias para dar respuesta a las necesidades del ser humano: la convivencia de la comunidad, el encuentro entre individuos o grupos, la escala humana y la identidad o sentido de pertenencia (fig. 02). A pesar de las alteraciones sufridas en el diseño original, la vigencia de estos conceptos teóricos permanece hoy en día en la ciudad construida.

En su definición, y a diferencia de los desarrollos urbanos tradicionales -de mayor compacidad en su centro-, Nagele estructuraba su traza en torno a un gran espacio verde; una pradera central como lugar de encuentro vecinal. Como límite, una barrera boscosa en su perímetro ofrecía a la ciudad un telón de fondo frente a las llanuras del pólder y funcionaba como cortavientos natural, protegiéndola climáticamente, convirtiendo Nagele en lo que Van Eyck denominó una habitación verde sin techo.

\subsection{Urbanismo Arquitectónico}

Para la definición del tejido urbano, Aldo van Eyck introdujo la configuración como herramienta de ordenación. ${ }^{3}$ Definió un cinturón de siete unidades residenciales encadenadas, cada una con sesenta viviendas. Las unidades presentaban un patrón de agrupación que no se repetía, sino que combinaba sus piezas con movimientos reflejados, giros y simetrías invertidas, siguiendo una disposición centrífuga en torno a un jardín o plaza arbolada.

Van Eyck tuvo la oportunidad de aplicar el mismo método de diseño a escala de edificio, en la construcción de tres escuelas para Nagele (1954-56). Tanto su proyecto para las escuelas como el planeamiento estaban diseñados de forma similar: un gran espacio público rodeado de elementos construidos que se enlazaban mediante plazas centrífugas cuadradas. Mientras que en el diseño del planeamiento las unidades repetidas eran bloques de viviendas, en las escuelas eran aulas; las plazas centrífugas eran vestíbulos de relación y el gran centro abierto el patio de juegos.
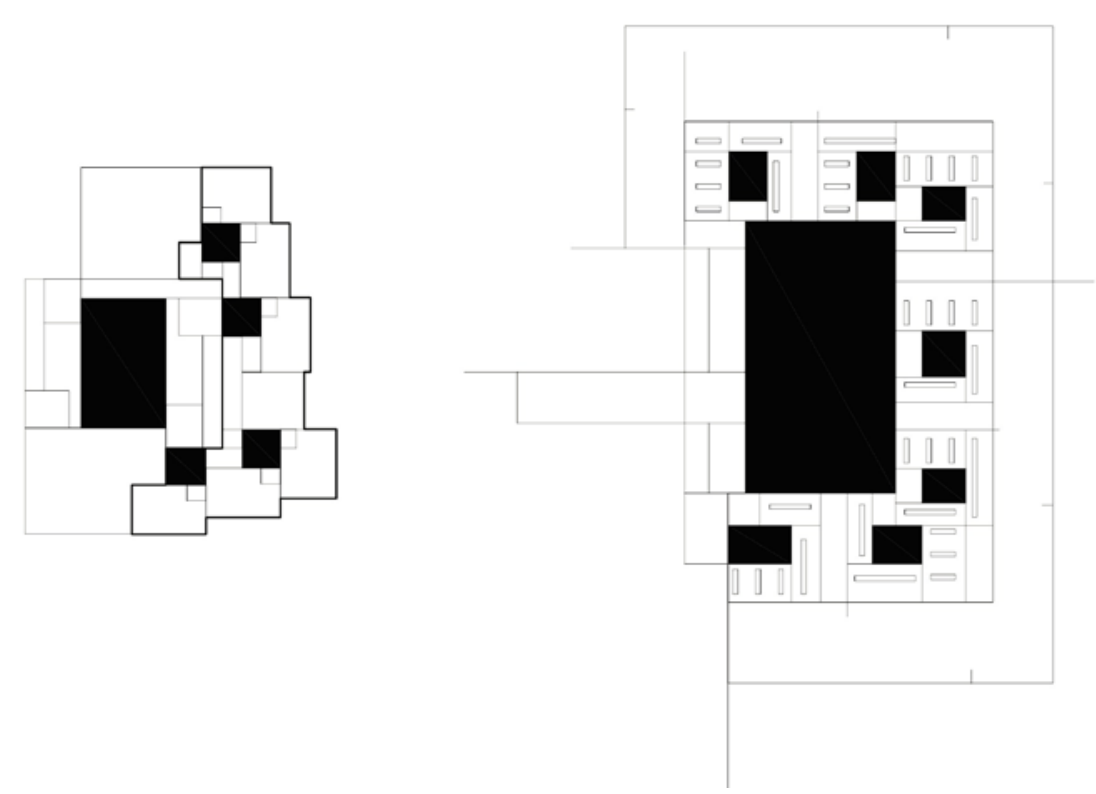

Fig.03 Analogía estructural parte-todo. Escuelas (izq.) y plan de Nagele (dcha.) Elaboración propia.

Aldo van Eyck encontraba una analogía estructural como herramienta para afrontar el diseño de la pequeña y la gran escala simultáneamente, mediante la vinculación recíproca entre ambas estructuras. Sin embargo, Van

${ }^{3}$ Ver: PALACIOS LABRADOR, Luis. 'Hacia un método de configuración'. Van Eyck / Blom / Hertzberger. Iniciadores y sucesores. Tesis Doctoral. Director: José Manuel López Peláez. Universidad Politécnica de Madrid, Departamentos de Proyectos, ETSAM, 2017. 
Eyck no fue el único que identificó la configuración del tejido urbano en la definición arquitectónica.

La más evidente era la réplica del modelo urbano que los arquitectos Mien Ruys, Wim Boer y Hendryk Hartsuyker hicieron para el diseño del cementerio de Nagele. En 1955, los primeros bocetos mostraban cómo una masa boscosa, equivalente al cinturón verde que envuelve la ciudad, definía los límites del cementerio, convirtiéndolo en un jardín cerrado, un hortus conclusus que enfatizaba la estructura autónoma y ensimismada del cementerio (fig. 04).

La organización interna también reflejaba un paralelismo con el planeamiento: un eje cubierto con una bóveda verde dividía el cementerio en dos ámbitos, al igual que lo hacía la carretera de acceso a Nagele, que separaba el asentamiento en dos, manteniendo la relación áurea entre ambas partes. ${ }^{4}$ En un estudio pormenorizado se comprueba cómo el patrón policéntrico de agrupación de hileras de tumbas era análogo al de las escuelas y las unidades residenciales. ${ }^{5}$
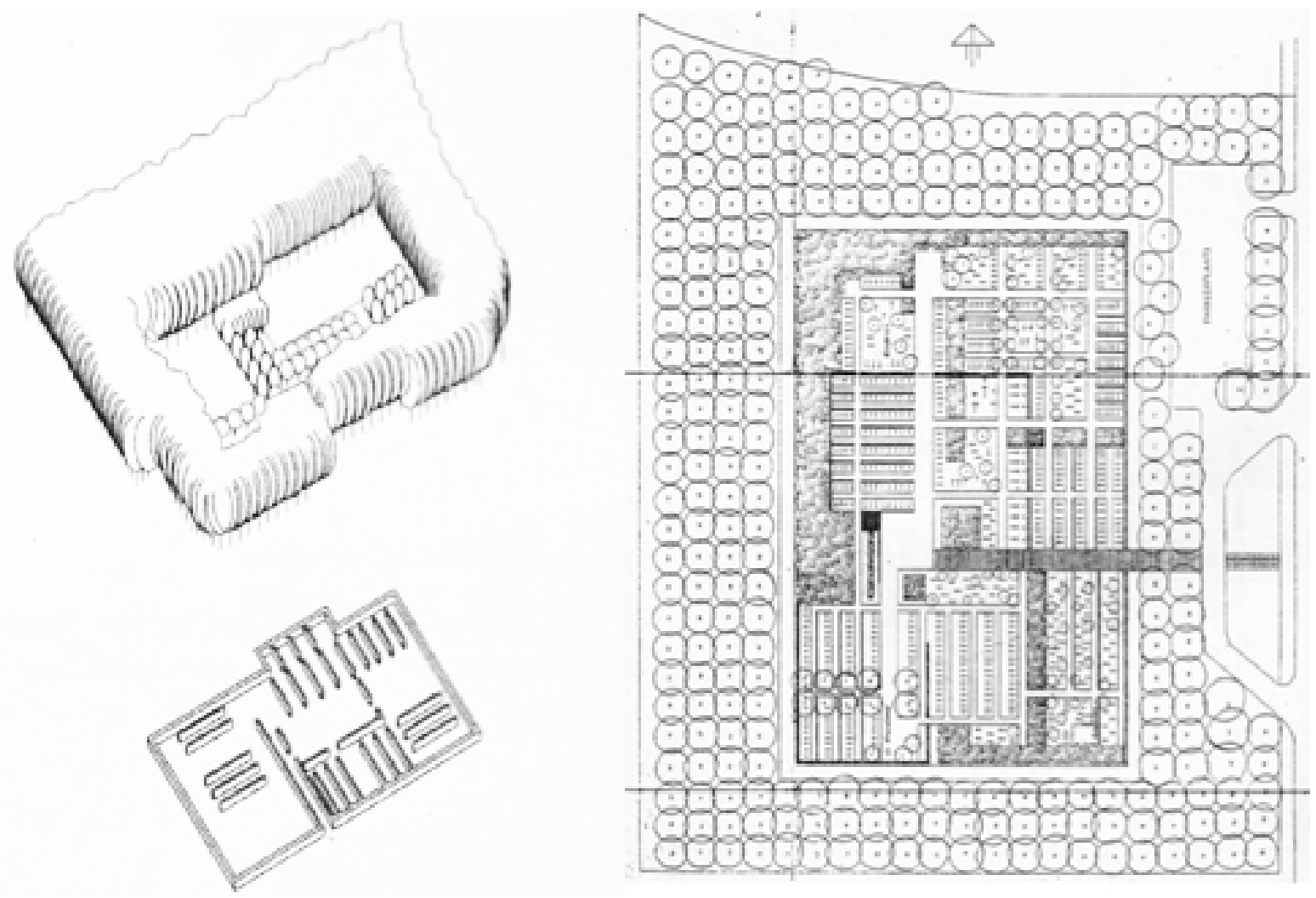

Fig.04 Cementerio de Nagele. Límites vegetales (izq. y centro) y organización de tumbas (dcha). Dibujos de Marta García Carbonero. Fuente: (CARBONERO, 2011)

De forma equivalente, el grupo De 8 en Opbouw, encargado de diseñar el programa residencial, replicó la estructura del planeamiento en cada cluster de viviendas. ${ }^{6}$ Independientemente del vecindario (Karweihof, Vlashof, Klaverhof, etc.) arquitecturas diseñadas en subgrupos de arquitectos como G. Rietveld, Lotte StamBesse o Stam y Van Bodegraven, organizaban las viviendas en hilera en torno a un centro abierto, como a su

\footnotetext{
${ }^{4}$ Las proporciones de Nagele, presentan una relación dimensional de 3 a 5, que puede encontrarse también en el cementerio. El eje de acceso cubierto por la bóveda verde, divide el cementerio en dos partes que muestran dimensiones relativas de 3 a 5 .

${ }^{5}$ Propuesta del 29 de octubre de 1955 que agrupaba seis unidades funerarias dispuestas alrededor de un espacio común, apareciendo a menor escala, el patrón policéntrico del asentamiento urbano global.

6 "La palabra 'cluster' significa un patrón especial de asociación y se introduce para reemplazar palabras como casa, calle, barrio o ciudad." Cita de los Smithson recogida en: NEWMAN, Oscar. CIAM'59 in Otterlo. Stuttgart: Karl Krämer, 1961, pp. 69.
} 
vez lo hacían los clusters alrededor de la pradera central de la ciudad.

El proyecto respondía así a un planteamiento fractal en el que el diseño de la parte se identificaba en el todo, y viceversa, unificando arquitectura y urbanismo en una sola disciplina: la arquitectura urbanística o el urbanismo arquitectónico. Pero esta analogía se llevó a los límites en el diseño de los edificios singulares de Nagele.

\subsection{Arquitectura Urbanística}

El proyecto de cafetería y el centro cívico (1957), fue desarrollado por J. Dunnebier, arquitecto que no formaba parte del equipo De 8 en Opbouw, y por tanto no había colaborado en los trabajos de ordenación urbana. Aún así el edificio, diseñado con una gran sencillez y sobriedad, envolvía con su programa más público (cafetería, restaurante, escenario y salón de actos) un vestíbulo de entrada análogo a la plaza de acceso a la ciudad, replicando su estructura a una escala menor (fig. 05).
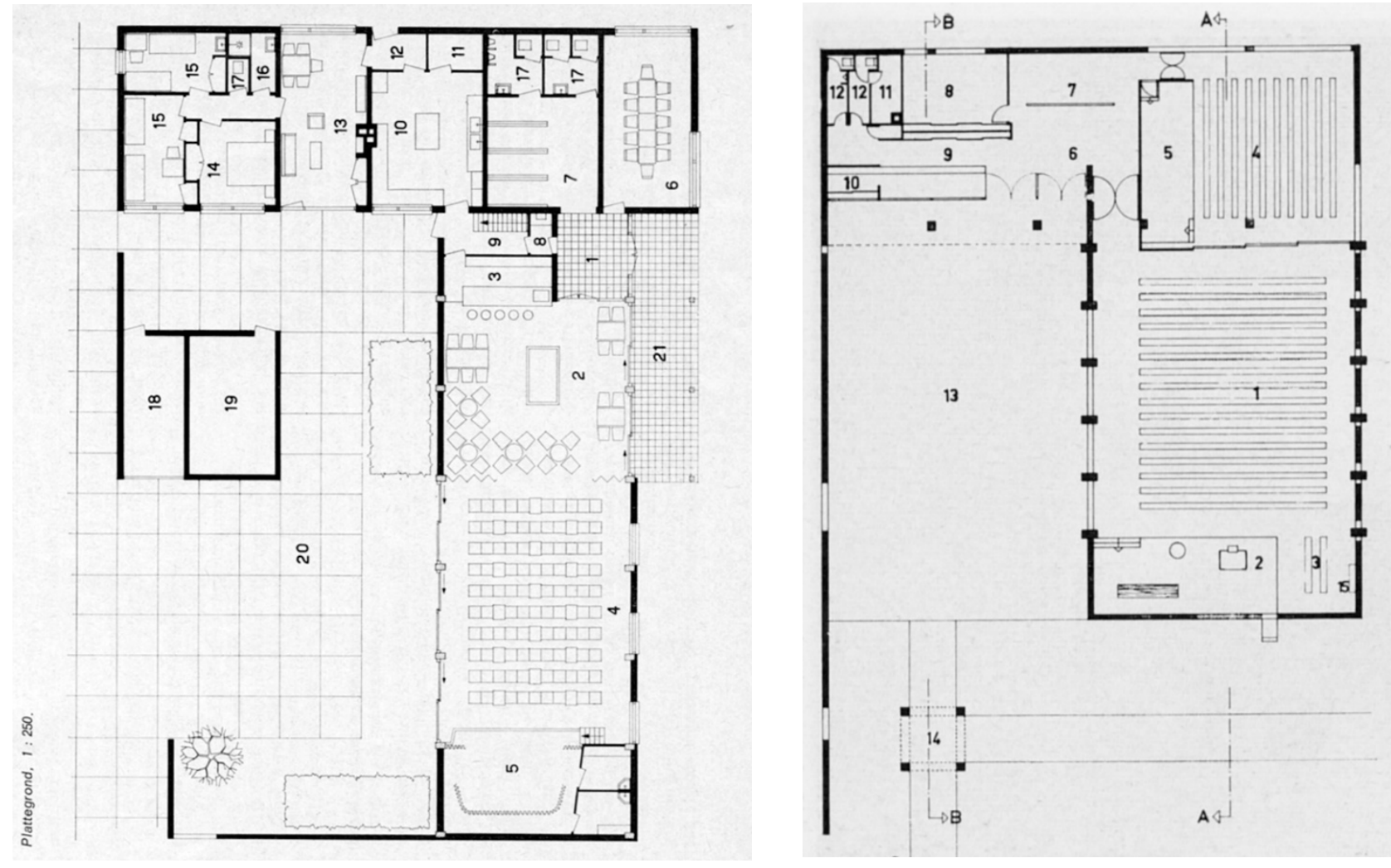

Fig. 05 (izq.) Plano en planta de la cafetería restaurante Schokkererf. Fuente: (DUNNEBIER, 1964)

Fig. 06 (dcha.) Planta de la iglesia. Fuente: (BAKEMA, 1961)

Más evidente es el proyecto de Iglesia de Jaap Bakema y Van der Broek, quienes participaron activamente en la segunda fase de planeamiento. La iglesia se ubica al noroeste de la pradera central, generando con el campanario un hito vertical que puede observarse desde la carretera regional. Detrás de la torre, un muro exterior va creciendo y replegándose, envolviendo un patio de entrada a la iglesia. Este hecho evidencia una analogía entre el muro que envuelve el edificio y el cinturón verde que rodea la ciudad (fig. 06).

Un estudio detallado descubre el reflejo de la estructura urbana incluso en el mobiliario litúrgico: un muro en espiral hace de púlpito de la iglesia que muestra, de nuevo y a escala menor, el concepto 'espacio dentro de otro espacio', de acuerdo al planteamiento fractal del proyecto. 


\section{El crecimiento de Nagele: de la utopía a la realidad}

\subsection{La ciudad terminada de Rietveld}

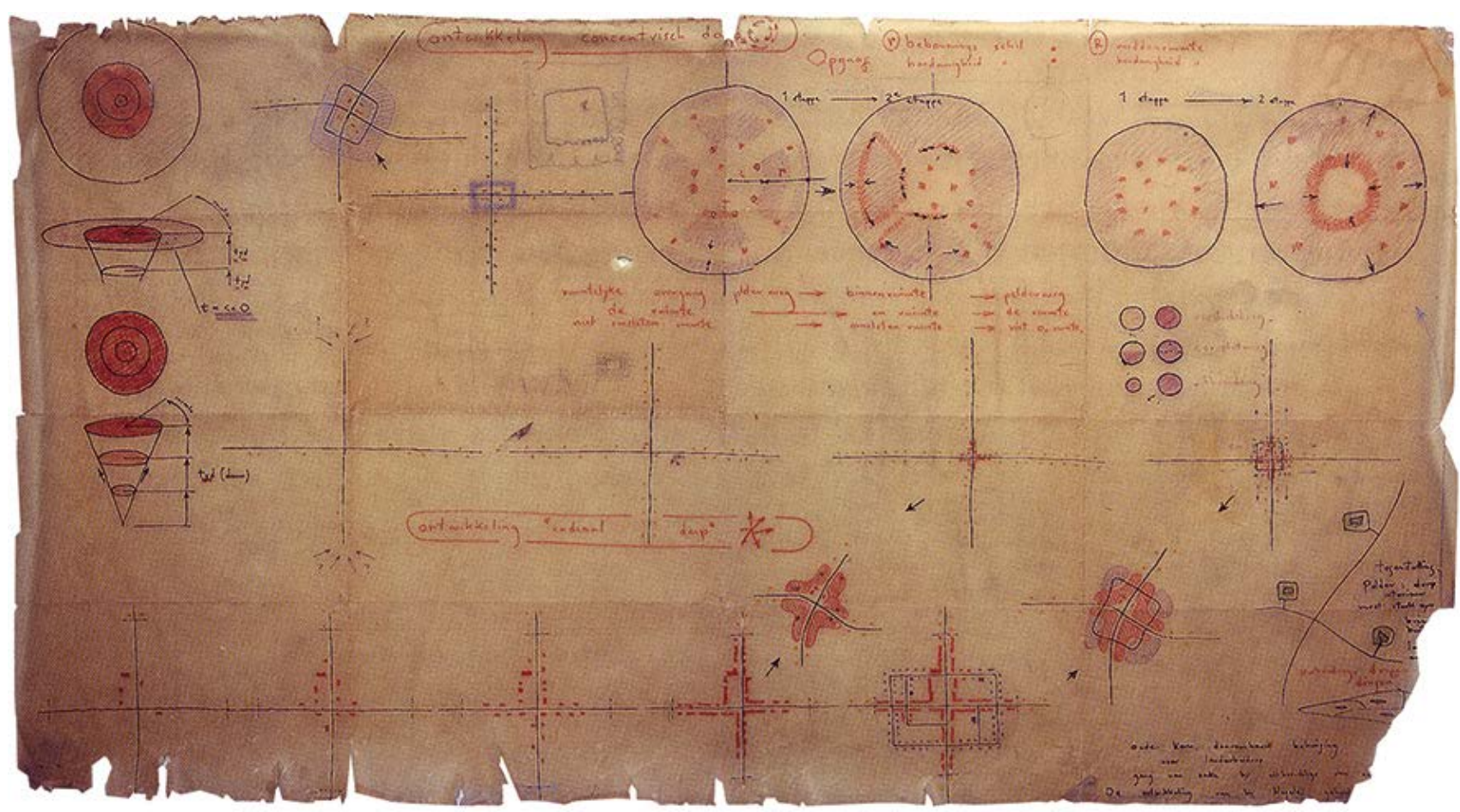

Fig. 07 Bocetos diagramáticos para Nagele. Van Eyck, reunión 14 de enero 48. Fuente: (STRAUVEN, 1994)

La visión holística del proyecto, donde arquitectura y urbanismo aparecen definidos por los mismos patrones de diseño, desde la ciudad a la silla, ${ }^{7}$ implicaba para el equipo redactor $D e$ en Opbouw el entendimiento de un proyecto cerrado, la utopía de una ciudad acabada desde su construcción, como anunciaba Gerrit Rietveld en un boceto a mano de Nagele:

"Un esfuerzo para hacer el pueblo de una sola vez, es decir, que no tenga un centro que vaya creciendo periféricamente, a base de construcciones. Éste es un pueblo que no crece, sino que se construye de una sola vez $[\ldots] "$

(Rietveld, 1948)

La reflexión de Rietveld muestra cómo una vez completado el cinturón del programa residencial previsto, el asentamiento urbano no debía seguir creciendo, porque se perdería la relación directa entre el conjunto de viviendas, de carácter privado, y el centro que representa la comunidad (fig. 08). La construcción de Nagele, planificada en dos fases, limitaba su expansión con la envoltura boscosa, que hacía del asentamiento una ciudad terminada desde su origen. (fig. 07).

\footnotetext{
${ }^{7}$ Referencia al texto de Bakema: "From Doorstep to Town”. Forum, no4, 1962, pp.127-131.
} 


\subsection{Expansión urbana controlada}

A pesar de la visión utópica de Gerrit Rietveld (fig. 08), la propuesta de expansión del asentamiento llegaría por parte de uno de los miembros del equipo De 8 en Opbouw. El arquitecto J.P. Kloos elaboró dos décadas después de su construcción, un plan de desarrollo para Nagele: la extensión de la ciudad hacia el este, más allá de la barrera boscosa, mediante una sistema de clusters de viviendas análogo al proyecto construido (fig. 09). La aproximación de Kloos suponía un crecimiento controlado que respetaba los principios configurativos inducidos por Van Eyck. A pesar de su interés, la propuesta no se llevó a cabo, dejando abierto el futuro de Nagele a diversas interpretaciones, algunas de ellas contrarias al concepto urbano original.
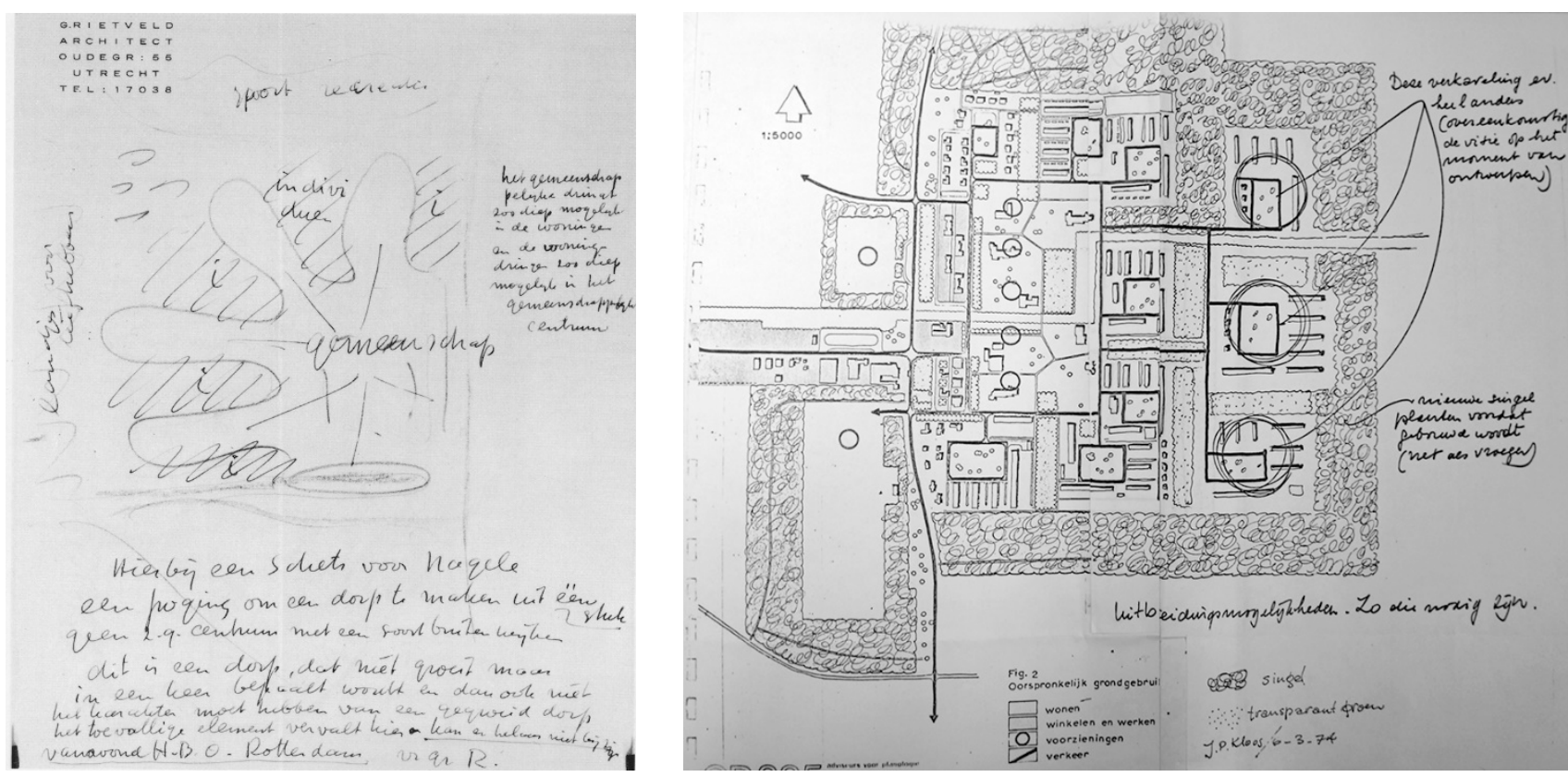

Fig. 08 Gerrit Rietveld, plano para Nagele, abril de 1948. Fuente: HEMEL Zef, VAN ROSSEM Vincent, op. cit., pág. 17. Fuente original: archivo Kamerling, NAi.

Fig. 09 J.P. Kloos. Proyecto de expansión de Nagele hacia el Este. 6 de marzo de 1974. Fuente: archivos NAi.

\subsection{Densificación y quiebra del prototipo}

Quince años después de la propuesta urbana de Kloos, un nueva alternativa llegaría de la mano del arquitecto Endry van Velzen (fig. 10). Basado en la densificación del asentamiento en lugar de su extensión, Van Velzen desarrolló un proyecto compuesto por elementos singulares que se insertaban en el tejido existente. Proponía viviendas de propiedad privada y pública, algunas de ellas para la tercera edad, así como la reorganización de los equipamientos comerciales y de servicios.

En primer lugar, incorporaba un cinturón de viviendas unifamiliares en el perímetro externo a la barrera boscosa, con tamaño determinado, pero con edificación libre, conectadas entre sí mediante un viario entre las parcelas y una hilera de arbolado que preservaba la silueta verde exterior de la ciudad. Este nuevo conjunto se articulaba con el asentamiento original a través de los corredores verdes compuestos por arboledas ordenadas, planificados por De 8 en Opbouw, que atravesaban la envoltura boscosa.

El proyecto de Van Velzen redefinía la zona de tiendas y negocios, manteniendo únicamente la cafetería y centro cívico por ser catalizador de la vida comunitaria. Agrupaba todos los equipamientos en bloques de 
orientación este-oeste, integrándose en las hileras de árboles existentes. La pradera central se densificaba con una serie de edificios residenciales idénticos de cinco plantas, localizados en posiciones estratégicas, conectados con los clusters pero sin obstaculizar la permeabilidad del tejido urbano. Su forma cilíndrica se liberaba de la condición ortogonal del planeamiento y aportaba mayor libertad en su ubicación, conectándose con las avenidas arboladas.

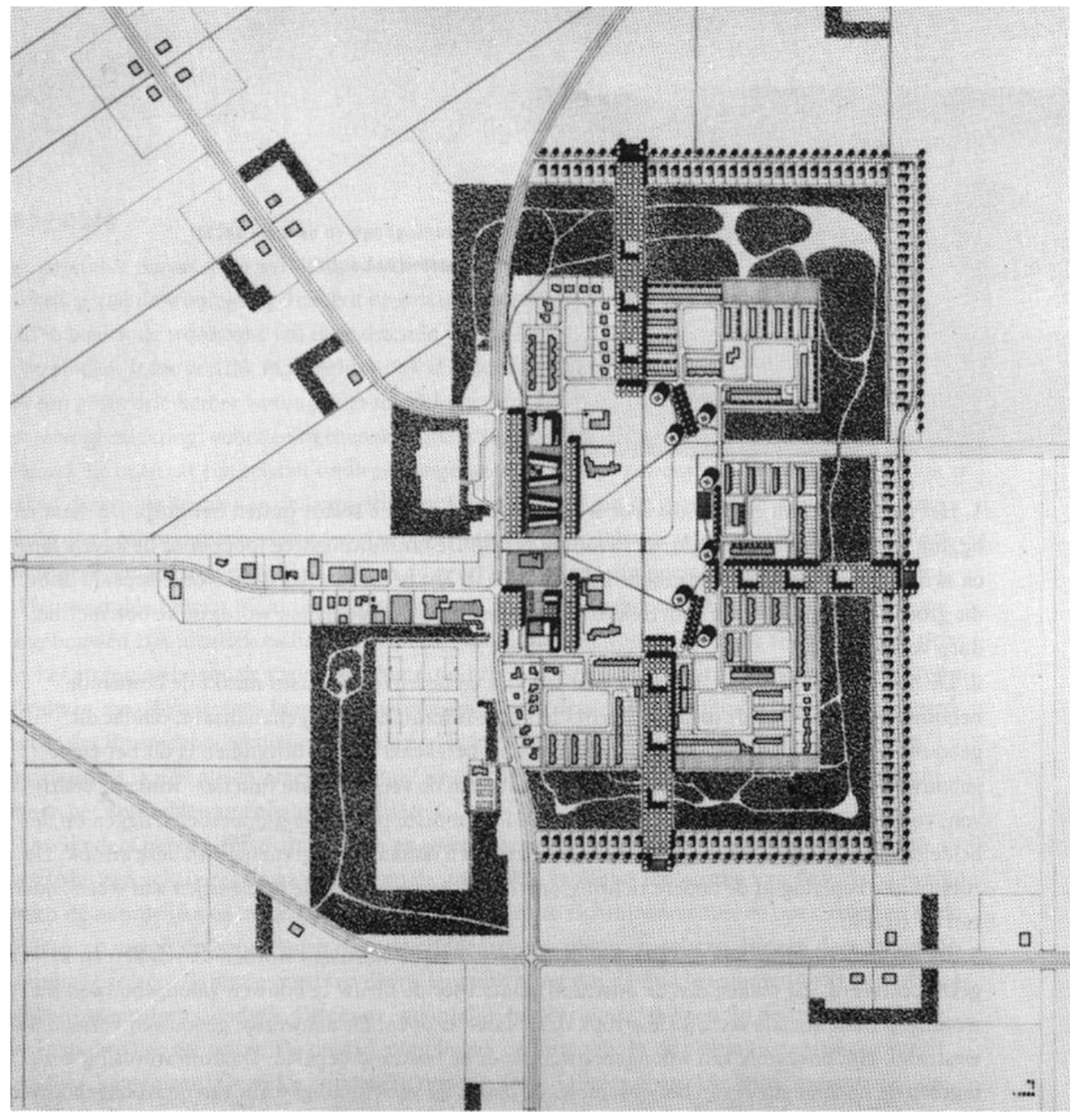

Fig. 10 Propuesta de expansión de Nagele, en la que se construye en la periferia urbana y en el centro colectivo. Fuente: (VAN VELZEN, 1989)

A pesar de la aportación de Van Velzen, el proyecto no fructificó y en los años 90 se aprobó el crecimiento de Nagele hacia el sur, medida que supuso la eliminación de la barrera boscosa y la construcción de dos nuevos clusters residenciales, Wendakkers y De Klamp, carentes de la estructura urbana del proyecto original. Sin atender al uso colectivo del espacio público ni a su vinculación con la pradera central, el nuevo desarrollo acogió a cien viviendas de propiedad privada. Ningún concepto de diseño definido por De 8 en Opbouw fue tenido en cuenta, a excepción de mantener la cubierta plana de la edificación. 
El proyecto construido, que llega a nuestros días desdibujando los principios configurativos del asentamiento original, nos invita a revisitar Nagele, ${ }^{8}$ analizando las debilidades que ha supuesto el paso del tiempo para encontrar líneas de actuación en el desarrollo de Nagele en el s. XXI.

\section{REVISITANDO NAGELE}

\subsection{Nagele hoy}

En la actualidad, el asentamiento de Nagele se enfrenta a una erosión de su base económica, la agricultura, que ha causado grandes cambios de largo alcance: migraciones, eliminación de servicios públicos, cierre de establecimientos, etc.

En consecuencia, la afección más evidente queda reflejada, más que en el núcleo urbano, en la unidad de gestión de los campos de cultivo, basada originalmente en una parcelación de 800×300 metros, malla productiva y sostenible debido a sus desplazamientos mínimos. Las granjas se incluyen dentro de las parcelas de cultivo, agrupándose en comunidades de dos, tres y cuatro unidades. Presentan una barrera boscosa contra el viento, abierta a la carretera y unas dimensiones idénticas.

Actualmente, los grandes avances en la mecanización y la falta de rentabilidad de la agricultura han propiciado la creación de monocultivos intensivos a gran escala, rompiendo el sistema equilibrado de granjas y parcelas de cultivo anexas. De las doscientas siete granjas que permanecen actualmente con función agrícola, en 2030 se espera una reducción a ochenta y tres. ${ }^{9}$ La evolución de la estructura de producción del campo requiere una reflexión sobre el tejido productivo del Noordoostpolder según las demandas agrícolas actuales, pero no es el único parámetro que debe ser revisado.

En Nagele, los espacios intermedios o in-between resultan estructurantes. Originalmente, la plaza de acceso era la puerta de entrada desde el pólder a la gran habitación verde sin techo. El muelle y el canal, pensados como una infraestructura de transporte fluvial, hoy se hallan en desuso, y el centro cívico demolido, por lo que este área demanda una nueva actividad.

Con la misma importancia, en cada cluster residencial, entre el viario en anillo y el patio residencial se define un ámbito intermedio en el que no se planificaron viviendas. Este umbral fue concebido como un lugar de encuentro para los residentes del vecindario con la comunidad. La idea original era ajardinarlos, o equiparlos con una dotación pública. Hoy en día se encuentran ocupados con pequeñas edificaciones privadas que disminuyen esta conexión.

De forma análoga, las zonas verdes actuales presentan serias alteraciones con respecto al diseño paisajista original. La extensión urbana hacia el sur supuso talar la barrera contra el viento original y plantar una nueva, considerablemente menos espesa. Las agrupaciones residenciales Koolzaadhof y Karweihof ya no se encuentran plenamente protegidas por la muralla boscosa, alejándose del concepto bunch of places de Van Eyck, que defendía en las ciudades una serie de espacios limitados y adaptados a la escala humana. La pradera central, originalmente entendida como una gran plano verde sobre el que se posan los equipamientos públicos, se ha ido ocupando con nuevos árboles no planificados, especialmente alrededor de los edificios, creando nuevos límites visuales anteriormente inexistentes. Un nuevo proyecto paisajista desarrollado por el bureau Mien Ruys ha comenzado a recuperar parte de las ideas del diseño original.

\footnotetext{
${ }^{8}$ Referencia al libro del fotógrafo BAART Theo, MARKERINK Cary, Nagele [revisited]. ed. NAi Publishers, Róterdam, 2006.

9 V.V.A.A, Werkboek Nagele. Projecten en uitwerkingen voor een modernistisch dorp in de polder, PHD Printmedia, Wormerveer, 2010. Proyecto número 14.
} 

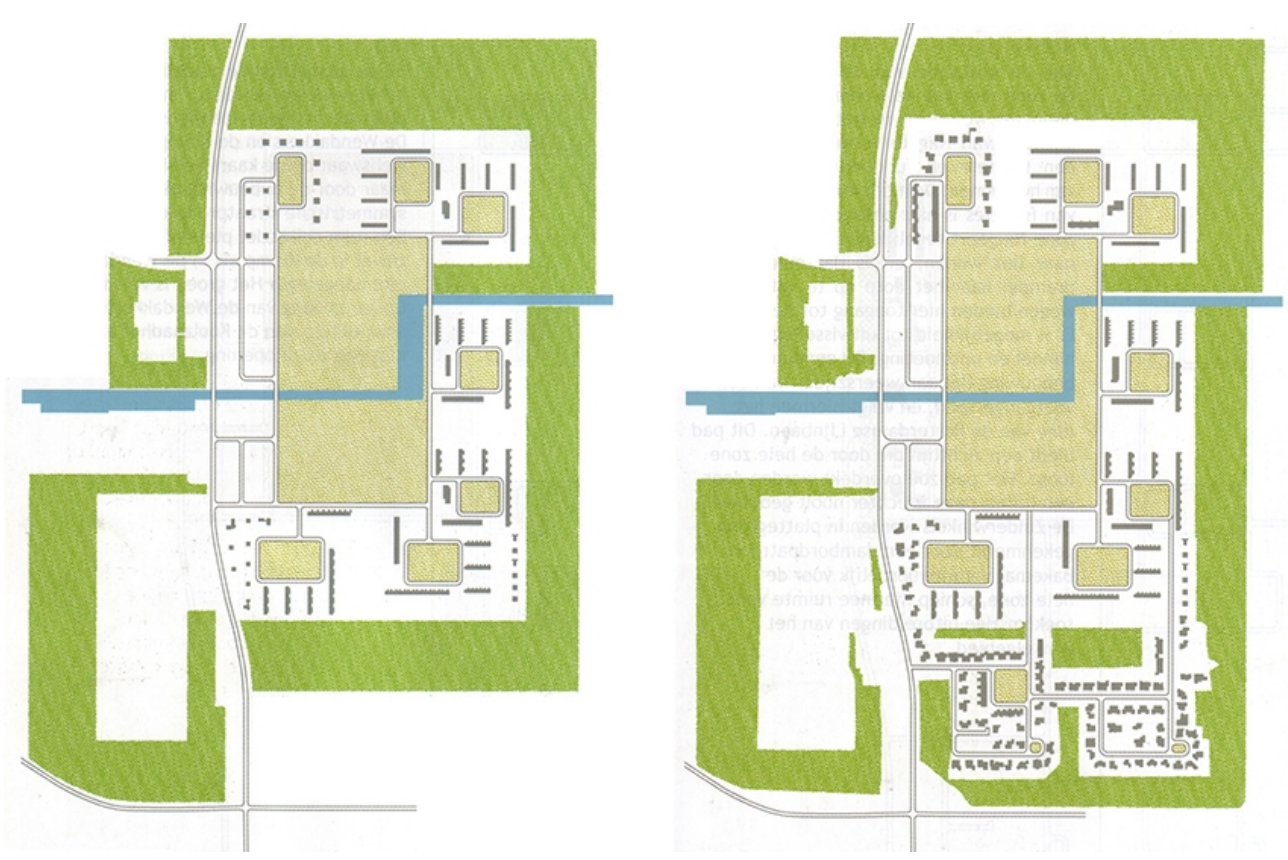

Fig. 11 A la izquierda, proyecto urbano desarrollado por De 8 en Opbouw en 1960. A la derecha, proyecto urbano en 2010. Fuente: (VV.AA., 2010, Handboek Nagele)

La gestión de lo construido también refleja cambios importantes, como el régimen de propiedad de las viviendas. Mientras que originariamente la mayor parte eran viviendas públicas, los habitantes han ido adquiriéndolas en propiedad. Este fenómeno ha causado operaciones de conservación no coordinadas que han afectado a la unidad del conjunto: diferencias de colores, materiales y cercas no planificadas perturban la legibilidad urbana. En este proceso, tal y como señala Van Gasteren, ha aumentado la condición de las viviendas como segunda residencia y carácter vacacional para holandeses de otras ciudades, o la inmigración procedente de países del Este. ${ }^{10}$

\subsection{Nagele Onderzoekslab}

La detallada explicación de la evolución histórica del planeamiento, junto con el análisis de la situación actual de Nagele, definen el marco de estudio del presente trabajo, completando el estado de la cuestión con investigaciones recientes sobre el futuro de Nagele.

Fue el gobierno holandés, a través del programa Nederland wordt anders, quien creó con interés equipos de trabajo para realizar estudios de mejora en diferentes emplazamientos de la nación. El intento de uno ellos, el Onderzoekslab Nagele, por mantener las ideas del proyecto original adaptándolas a las nuevas realidades económicas y sociales, fructificó en 2010 con dos publicaciones que recogen gran cantidad de análisis y propuestas, entre las que destacamos dos, por su aproximación a la escala urbana y regional. ${ }^{11}$

\footnotetext{
10 Un reflejo preciso del nuevo modelo son los documentales del artista Louis van Gasteren, mostrando las diferencias entre la vida en Nagele en el año 1960 (Película: Un pueblo en tierra nueva) y 2011 (Película: De vuelta a Nagele). Es, a partir del entendimiento de los cambios mencionados, donde aparecen líneas de trabajo que reinterpretan el tejido de Nagele, objeto de interés de esta investigación.

${ }^{11}$ Onderzoekslab Nagele estaba compuesto por un grupo de quince arquitectos jóvenes y liderado por dos mentores especializados, Pi de Bruijn y Bas Horsting, desarrollaron durante tres meses una serie de investigaciones analíticas y propositivas para la mejora de Nagele.
} 

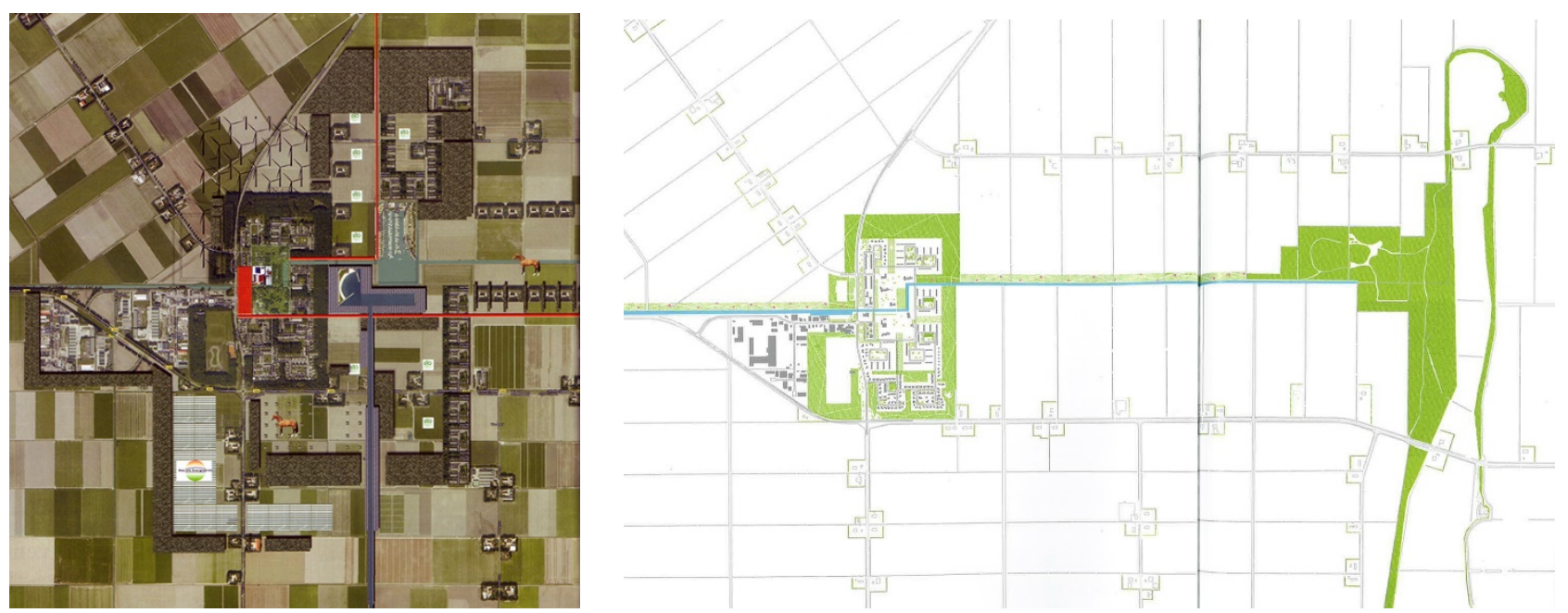

Fig. 12 Propuestas de futuro para Nagele. Fuente: (VV.AA., 2010, Werkboek Nagele)

La primera propuesta consiste en expandir la envoltura boscosa de la ciudad al pólder, replicándola a diversas escalas relacionadas mediante proporciones áureas que respetan el sistema de parcelación original. Protegidos por las nuevas barreras verdes, se definen recintos que albergan programa adaptado al siglo XXI, como nuevas agrupaciones residenciales, granjas e invernaderos a gran escala, cambiando el sistema de producción de la agricultura tradicional a la nueva economía basada en la generación de energía verde, turismo cultural, etc.

La segunda investigación aborda el paisaje territorial del Noordoostpolder, de fuerte valor histórico-cultural. El prototipo moderno de Nagele, contrasta con el patrimonio centenario de la isla de Schokland que quedó integrada en el diseño regional del pólder. Lo antiguo y lo nuevo son dos fenómenos que se han entendido de forma antagónica pero que pueden alcanzar un acuerdo en el territorio, un nuevo twin phenomena. ${ }^{12}$

Con este objetivo, el proyecto propone aumentar el valor experiencial del paisaje potenciando el eje del canal que une Schokland con Nagele. Un nuevo desarrollo urbano lineal genera un conjunto de arquitectura y naturaleza que fomenta, a través de una banda recreativa, el turismo rural, los deportes acuáticos, paseos en bicicleta, excursiones a pie, etc.

Pequeñas edificaciones residenciales en altura, separadas un mínimo de cincuenta metros para disminuir su influencia visual, proporcionan libertad formal al conjunto. Los tipos residenciales son flexibles y adaptables a la heterogeneidad de sus ocupantes. Toda construcción se inserta en un conjunto arbolado que oculta las viviendas desde la lejanía, reinterpretando la protección climática del proyecto original.

Ambas aproximaciones, de jóvenes arquitectos holandeses, motivan una investigación desde la distancia, para abordar la regeneración de Nagele desde una perspectiva académica que vaya más allá de los condicionantes propios del tiempo y el lugar.

Fuente: V.V.A.A, Werkboek Nagele. Projecten en uitwerkingen voor een modernistisch dorp in de polder, PHD Printmedia, Wormerveer, 2010. V.V.A.A, Handboek Nagele. Visie op een modernistisch dorp in de polder, RDH Printmedia, Wormerveer, 2010.

${ }^{12}$ Para Van Eyck, el concepto 'twin phenomena' proviene de comprender que las polaridades (abierto-cerrado, grande-pequeño, exteriorinterior) no se excluyen, sino que se complementan, como dos mitades de un todo. 


\subsection{Sobrescribir en la utopía}

"Las mejores contribuciones para dar respuesta a "el gran número" han sido hechas por mis estudiantes. Yo sé sobre ello, hablo de ello, leo sobre ello, pero es responsabilidad de las nuevas generaciones. Ellos serán los que encontrarán la llave."13

Con esta declaración, Aldo van Eyck delegaba el desarrollo de la disciplina de configuración a sus sucesores, la nueva generación de estudiantes de arquitectura. Esta intención nos mueve a concluir el presente texto reflejando una experiencia docente: la elaboración de un curso de proyectos en la Escuela de Arquitectura de Madrid (ETSAM, Universidad Politécnica Madrid) vinculado al Grupo de Investigación Cultura del Hábitat, donde proponemos una reflexión sobre Nagele como patrimonio del movimiento moderno de la posguerra.

El alumno debe revisar el asentamiento original para mantenerlo como fue pensado o reinterpretarlo, abordando simultáneamente la escala de la arquitectura y el urbanismo para proponer su densificación, crecimiento o revitalización del tejido urbano existente. Se recogen trabajos destacados como ejemplos representativos del desarrollo de curso:

Dada la necesidad de incrementar la escala de productividad agrícola, algunas propuestas se basan en la generación de sistemas estructurales de invernaderos y parques tecnológicos de industrias agroalimentarias que integran equipamientos, dotaciones e infraestructuras para la movilidad peatonal y el transporte de mercancías. Modelos contemporáneos de agricultural cities renuevan el debate sobre la limitación de la escala arquitectónica, sobrepasando los límites de la ciudad existente hasta llegar a los asentamientos colindantes, 0 incluso a la totalidad del pólder en una estrategia holística que abarca arquitectura, urbanismo y paisajismo.
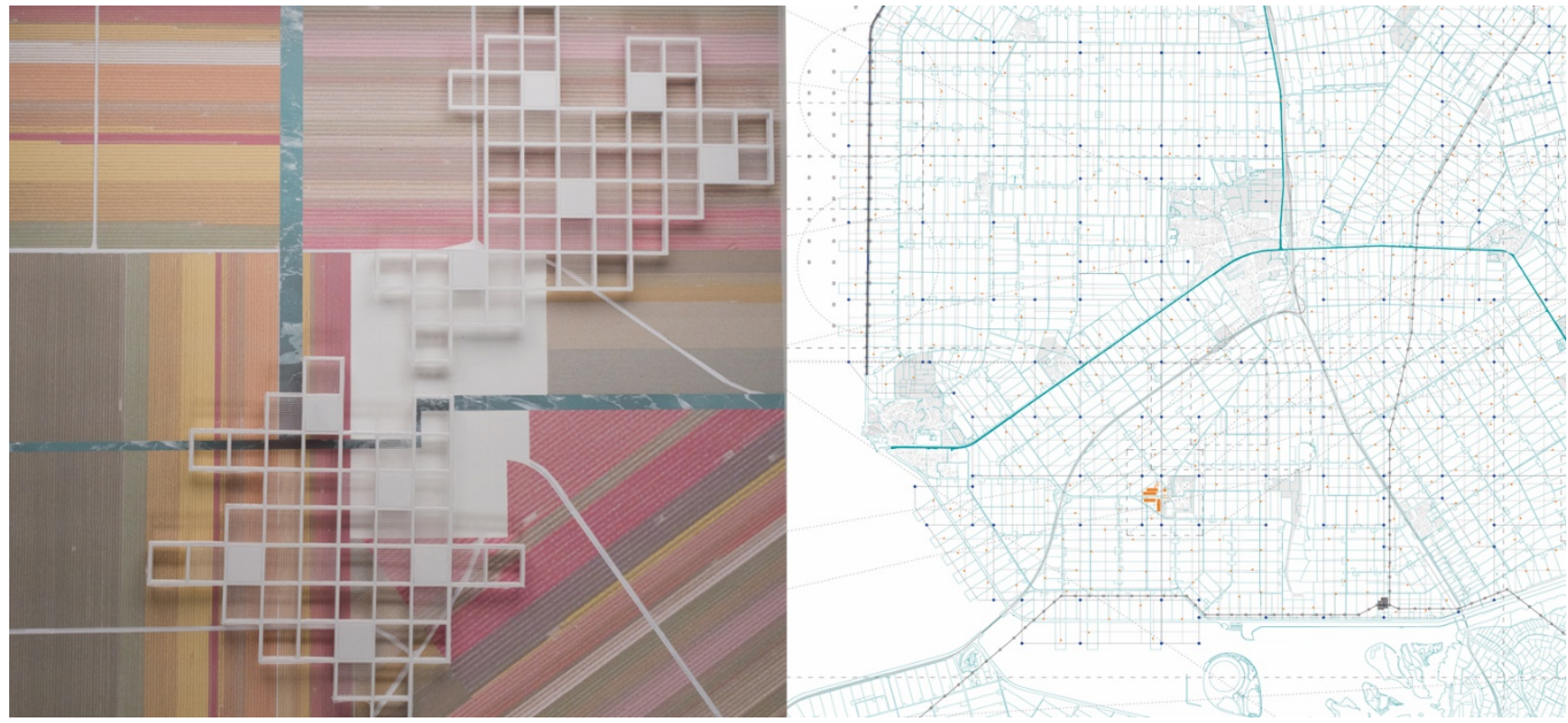

Fig. 13 Ejercicio de Claudia Baquedano y Ana Gutiérrez (izq.) Propuesta territorial de Beatriz Alonso y Francisco Balado (dcha.)

Ciertas aproximaciones recuperan el patrón de configuración del planeamiento original de Van Eyck, reinterpretando la idea de cluster residencial como soporte para el futuro crecimiento de la ciudad. Como investigación equivalente, reinterpretan la condición fractal de la estructura urbana -una habitación verde sin techo- para replicarla de forma análoga a todas las escalas.

${ }^{13}$ Extracto de la charla que Aldo van Eyck dio en la asociación australiana de estudiantes de arquitectura en Perth, Mayo 1966. En Strauven, Francis; Ligtelijn, Vincent: Collected Articles and Other Writings 1947-1998. Amsterdam: SUN Publishers, 2008, pp. 445. 


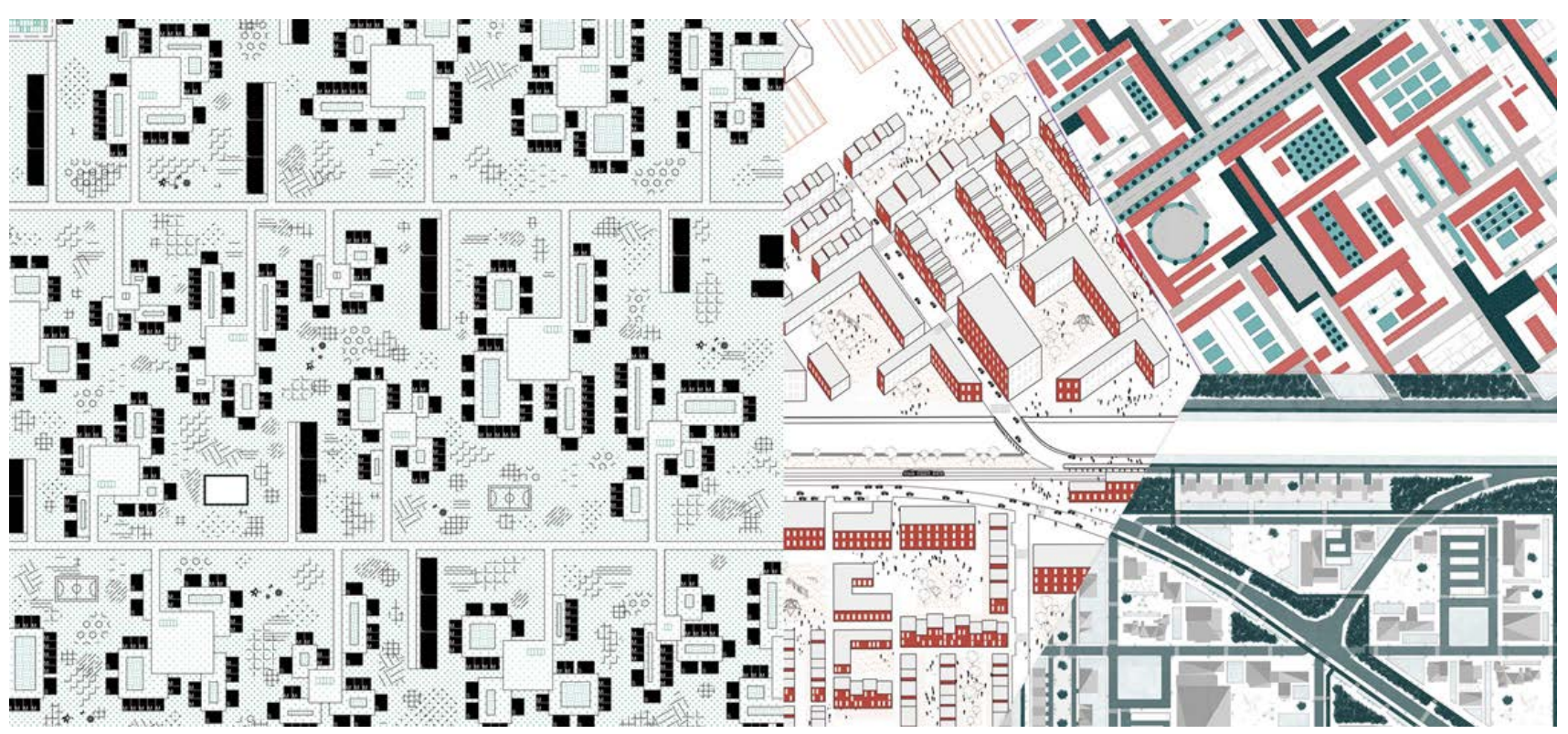

Fig. 14 Ejercicio académico de Ana María Diezma (izq.). Trabajo en grupo de Ismael Casado, Eduardo Schiebek y Sofía Vázquez (dcha.)

Por el contrario otros proyectos, en lugar de extenderse hasta el infinito, intervienen en el planeamiento original, densificando el tejido existente y dando respuesta al in-between realm o espacio intermedio, obsesión en el discurso teórico de Van Eyck. Colmatando los ejes verdes centrífugos que parten desde la pradera central de la ciudad o construyendo el camino del perímetro boscoso, se desarrollan proyectos que inciden en la concepción unitaria de arquitectura y paisaje, garantizando condiciones cualitativas de diversidad, porosidad y mezcla de funciones como alternativa a la monotonía unifuncional del tejido actual.
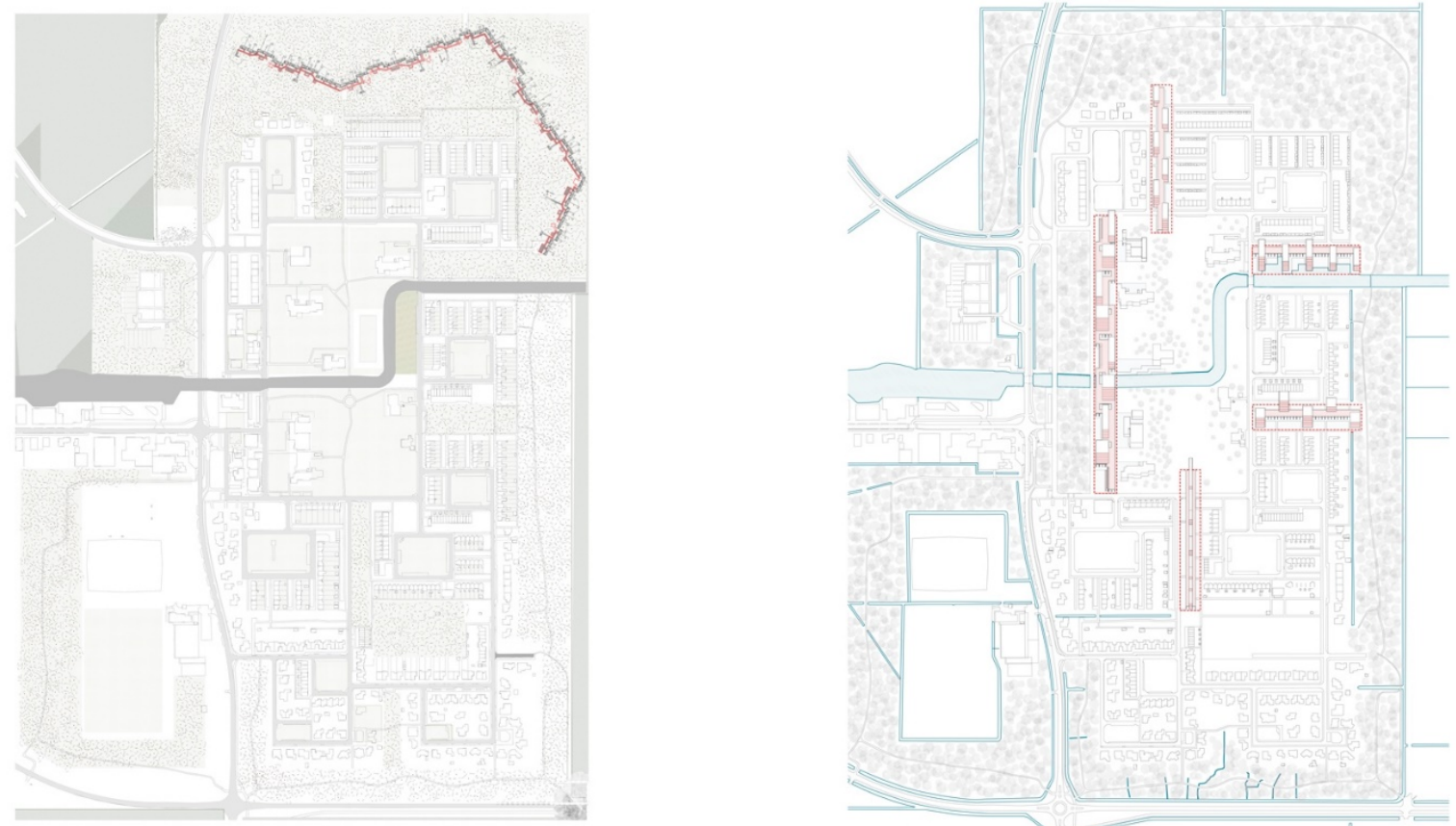

Fig. 15 Ejercicios académicos de Marta Navarro (izq.) y Nicoló Franchetto (dcha.) 
Como conclusión del presente trabajo de investigación, se destaca la reinterpretación de los proyectos académicos expuestos como reflejo de una mirada crítica y necesaria al asentamiento actual de Nagele, cuya estructura ya suponía una revisión a la ciudad moderna pero que ha quedado obsoleta por el cambio de paradigma social, económico y productivo.

Los planteamientos heterogéneos de estos jóvenes estudiantes vuelven a convertir el proceso de diseño en un interesante laboratorio de ideas donde se aplican múltiples conceptos teóricos en revisión y nuevas formas de proyectar la ciudad del siglo XXI. En sus planteamientos, han descubierto cualidades que no sólo ponen en valor los conceptos urbanos de Aldo van Eyck, sino que se muestran vigentes para su aplicación en el diseño de la ciudad contemporánea.

Siguiendo estas líneas de evolución, se abre un nuevo campo de estudio entorno al concepto de arquitectura urbanística o urbanismo arquitectónico, conceptos aparentemente contrarios que podrían reconciliarse reforzando, desarrollando y enriqueciendo las respuestas a la ciudad contemporánea en futuras investigaciones.

\section{BIBLIOGRAFÍA}

ABAD MONLLOR, E. (2016) Nagele: un nuevo asentamiento en un paisaje artificial. Tesis Doctoral. Universidad Politécnica de Madrid, Departamentos de Proyectos, ETSAM.

ANDELA G. (1982). Lusthof voor het nieuwe bouwen. Futura, 17, págs. 2-23.

ATEN D. et al. (2007). The sea of land. The polder as an atlas of Dutch landscape architecture. Ámsterdam: Architectura \& Natura.

BAART T., MARKERINK C. (1986). Nagele N.O.P. Ámsterdam: Fragment.

BAART T., MARKERINK C. (2006). Nagele [revisited]. Róterdam: NAi Publishers.

BASTA Urbanism, Bureau Mien Ruys (2012). Uitvoeringslab Nagele. Groenstructuurplan Nagele, ed. Gemeente Noordoostpolder y Rijksdienst voor het Cultureel Erfgoed (Ministerie van Onderwijs, Cultuur en Wetenschap). Noordoostpolder.

CONSTANDSE, A.K., VISSER, E.A. (1964). Een experiment getoetst. Het dorp met glamour. Bouw, págs. 1134-1139.

GARCÍA CARBONERO, M. (2011). Espacio, paisaje y rito: formas de sacralización del territorio en el cementerio europeo del siglo XX (tesis doctoral dirigida por Miguel Ángel Aníbarro). UPM ETSAM

HEMEL Z., VAN ROSSEM V. (1984). Nagele, een collectief ontwerp 1947-1957. Ámsterdam: Hoekstra bv te Emmeloord.

KEGEL R. (1996). De onderste laag boven: compositorische kenmerken van Pendrecht en Nagele. Delft: Publikatieburo Bouwkunde, Faculteit der Bouwkunde, Technische Universiteit.

NIJHUIS S. et al. (2010). Polder Atlas of the Netherlands. Pantheon of the low lands. Bussum: Thot.

PALACIOS LABRADOR, L. (2017) Hacia un método de configuración. Van Eyck / Blom / Hertzberger. Iniciadores y sucesores. Tesis Doctoral. Universidad Politécnica de Madrid, Departamentos de Proyectos, ETSAM. 
SAAL C.D. (1986). Een bijzonder dorp, dat is het en dat blijt het. De glamour van Nagele (NOP) opnieuw beschouwd. 50 jaar actief achter de Afsluitdijk. Jubileumbundel (...) Publikaties van de Stichting voor het Bevolkingsonderzoek in de drooggelegde Zuiderzeepolders (Zutphen), 85-118.

STEENHUIS M. (2009). Landscape for the advanced. Design and Development of the Noordoostpolder, 19422009. Oase (Róterdam), 80, 36-48.

STRAUVEN F. (1994). Aldo van Eyck, the shape of relativity. Ámsterdam: Architectura \& Natura.

STRAUVEN, F., LIGTELIJN, V. (2008). Collected Articles and Other Writings 1947-1998. Amsterdam: SUN Publishers.

VAN BODEGRAVEN W.F. et al. (1952). Een plan voor het dorp Nagele. Forum (Ámsterdam), 6-7, 172-178.

VAN DER WAL C. (1997). In praise of common sense. Planning the ordinary. A physical planning history of the new towns of the ljsselmeerpolders. Róterdam: 010 Publishers.

VAN EYCK, A. (1960) Three Schools in Nagele. Architects' Year Book, nº 9, 140-143.

VAN EYCK, A. (1962) Steps towards a configurative discipline. Forum, no 3, 81-94.

VAN GASTEREN L. (2011). Terug naar Nagele [DVD]. Amsterdam: Spectrum Film.

VAN GASTEREN L. (1960). Een nieuw dorp op nieuw land [DVD]. Ámsterdam: Spectrum film.

VAN VELZEN, E. (1989). Een uitbreidingsplan voor Nagele. Oase (Róterdam), 26 y 27, 78-84.

V.V.A.A. (2010). Werkboek Nagele. Projecten en uitwerkingen voor een modernistisch dorp in de polder. Wormerveer: RDH Printmedia.

V.V.A.A. (2010). Handboek Nagele. Visie op een modernistisch dorp in de polder. Wormerveer: RDH Printmedia. 